\title{
USING THEORETICAL-COMPUTATIONAL CONFLICTS TO ENRICH THE CONCEPT IMAGE OF DERIVATIVE
}

\author{
Victor Giraldo, Universidade Federal do Rio de Janeiro, Brazil \\ David Tall, University of Warwick, United Kingdom \\ Luiz Mariano Carvalho, Universidade do Estado do Rio de Janeiro, Brazil
}

Recent literature has pointed out pedagogical obstacles associated with the use of computational environments in the learning of mathematics. In this paper, we focus on the pedagogical role of computer's inherent limitations on the development of learners' concept images of derivative and limit. In particular, we intend to discuss how the approach to these concepts can be properly designed to prompt a positive conversion of those limitations to the enrichment of concept images. We present results of a case study with six undergraduate students in Brazil, dealing with situation of theoretical-computational conflict.

\section{INTRODUCTION}

A wide range of issues related to the use of technology on the teaching of Mathematics has been discussed in the literature. Some of those research works report experiments in which a narrowing effect takes place: limitations of the computational representation lead to the development of restricted concept images by learners (e.g. Hunter, Monaghan and Roper, 1993). The aim of this research is to discuss how the pedagogical role of those limitations can be positively converted to enrich students' concept image of derivative and limit. We have defined theoretical-computational conflict to be any situation in which a computational representation for a mathematical concept is (at least potentially) contradictory with the associated theoretical formulation (Giraldo, 2001; Giraldo and Carvalho, 2002). For example, numerical calculation with machine accuracy cannot be performed in a way that corresponds exactly to the mathematical theory of limits. Our hypothesis is that, if theoretical-computational conflicts are emphasized, rather than avoided, they may contribute not to narrow, but to enrich, concept images. In this paper, we will present some results of an experiment, in which we have observed a sample of six undergraduates students, from a Brazilian university, dealing with theoretical-computational conflict situations.

\section{CONCEPT IMAGES AND COGNITIVE UNITS}

In the theory developed by Tall and Vinner (1981), the concept image is the total cognitive structure associated to a mathematical concept in an individual's mind. It includes all the mental pictures, properties, mental associations and processes related to a given concept, and is continually constructed as the individual matures, changing with new stimuli and all kinds of experiences. Moreover, the concept image may (or not) be associated to a statement used to specify that concept, named the concept definition by the authors. A concept definition, in its turn, may (or not) be coherent 
with the formal mathematical definition (that is, the concept definition usually accepted by the mathematical community). Thus, the individual's concept image may or not include the formally correct definition (see also Vinner 1983,1991; Tall, 2000).

Tall and Barnard (1997) have named as cognitive unit each chunk of the concept image in which an individual can focus attention at a time. Cognitive units may be symbols, theorems, representations, properties or any other aspects related to the concept. The authors claim that the ability to build multiple and flexible connections between cognitive units and within themselves, and therefore to reach important information whenever necessary, is an important feature to the development of powerful mathematical thinking. Thurston (1990) affirms that human strategies to understand mathematics often demand a process of mental compression, in which ideas can be quickly and completely recalled. In this way, a rich concept image must include, not only the formal definition, but a large number of linkages both within cognitive units and between them.

\section{NEGATIVE EFFECTS OF THE USE OF COMPUTERS ON MATHEMATICS TEACHING: NARROWING CONCEPT IMAGES}

In this investigation, we intend to focus on the positive use of technology in mathematics learning. However, it is important to remark that research shows that misused computational environments can have negative (or at least unhelpful) effects in mathematics education. The theory quoted above suggests, in particular, that teaching the concept of derivative must comprise different approaches and representations, to enable learners to build up multiple and flexible connections of cognitive units and, therefore, a rich concept image. Each representation gives emphasis to certain aspects of the concept, but also blots out others. Tall (2000) affirms that the focus on certain aspects and the negligence of others may result in the atrophy of neglected ones. Literature provides some examples in which that process takes place on computerbased approaches.

An instance is found in Hunter et al. (1993). The authors observed that students using software Derive in computer-based instruction did not need to substitute values to get a table and sketch the graph. As an unforeseen consequence, some students who could calculate values by substitution before the course seemed to have lost the skill afterwards. Before and after the experiment, a sample of seventeen students was asked:

What can you say about $u$ if $u=v+3$ and $v=1$ ?

None of the students who had failed on the pre-test improved on the post-test and, moreover, six who had given satisfactory answers on the pre-test failed on the posttest.

Results of recent research developed in Brazil also reveal difficulties of students and teachers about the use of new technologies. Abrahão (1998) observed the reactions of four secondary teachers dealing with graphs of functions produced by computers and graphic calculators. In the activities applied, results given by the devices seemed to 
contradict the mathematical theory, due to software limitations or inadequacy of visualization windows. In the course of the experiment, the teachers hesitated to take into consideration the fact that computers can provide "mistaken" or "incomplete" results. In fact, those results were often accepted by participants as correct without query, even when clearly clashing to their prior knowledge. The author comments:

All the teachers, even the ones who knew the computers had limitations, seemed, initially, to believe in the computer results, rather than in their own knowledge. [...]

When asked to interpret some unusual graphs produced by computers, the interviewees had some difficulties. We observed that the comprehension of graphs generated by technology is not immediate. Teachers did not always manage to conciliate their knowledge with the visualization on screen. (Abrahão, 1998, p. 23, our translation)

Similar results are reported by Belfort and Guimarães (1998). The authors observed teachers' behaviour in the course of activities on dynamic geometry, using the software SketchPad. On one of the activities, participants were asked to find empirically the rectangle with perimeter $40 \mathrm{~m}$ and largest area possible. The software allowed users to construct a rectangle, gradually varying the sides' measures, keeping the perimeter fixed, and observe the consequent variation of the area. Due to floating point arithmetic, the software could only generate approximate results. For example, one of the teachers found a rectangle with area $100 \mathrm{~m}^{2}$, sides $10.03 \mathrm{~m}$ and $9.97 \mathrm{~m}$. He then showed great confusion: he could not cope with such a mismatch at all. The authors comment:

[...] many teachers accepted the result given by the software as conclusive. In one of the experiments, we were requested by three groups of teachers [each one consisting of two members] which, seating nearby to each other, had compared their results. All of them had obtained the maximum area of $100 \mathrm{~m}^{2}$, but the values for the side $A B$ were different. They had ended up in a deadlock, and were unable to figure out which of the three values would be the correct one. The investigation about the software 'mistake' led to the necessity of finding a theoretical solution for the problem. (Belfort and Guimarães, 1998, p. 5, our translation.)

Laudares and Lachini (2000) observed the process of introduction of a computer laboratory for the teaching of Calculus (LABCAL) in a large Brazilian university, which had been following a traditional approach before. The interviews with the Calculus teachers showed that most of them believed that laboratory activities would be a waste of time, which should be spent with classroom instruction, and that the use of computer should be restricted to very complex calculations. Observing the activities in the laboratory, the authors comment:

One student, who works alone, types the data of the problem, strikes the keys for the resolution and reads the answer on the screen. Since he does not have knowledge of the mathematical subject, he doesn't know if the answer is right. He then checks the answer with a classmate and, without any query, proceeds to the resolution of the next exercise. He has great difficulty, not only with the manipulation of the device, but also 
does not seem to understand the meaning of the results given by the computer. For two students who work together, the very first difficulty is to access the system. [...] One of them types the data of the problems, meanwhile the other one just follows the activity. When requested to clarify doubts, the instructor restricts himself to commenting: "the theory should be learnt in the classroom; the activity in LABCAL is only to calculate, using the keys correctly'. Despite the fact that they talk considerably about the activity, the students do not seem to realise the aims of what they are doing. (Laudares and Lachini, 2000, pp. 5-6, our translation.)

The authors conclude that the use of technology can constitute an important alternative for the traditional model of lecture, however, in designing computer activities, it is necessary to work towards the development of a critical and investigative perspective by students.

\section{USING THEORETICAL-COMPUTATIONAL CONFLICTS TO ENRICH CONCEPT IMAGES}

Research quoted in the preceding section shows the negative effects caused by the misuse of computational environments in learning mathematical concepts. The experiment reported by Hunter et al., in particular, has uncovered a narrowing effect on concept images: the intrinsic characteristics of the computational representation led to limitations in the concept images developed by learners.

Many authors agree that the effects of computers on mathematics learning does not depend on the devices themselves, but on the way they are (mis)used. Tall remarks that:

... the use of calculators and computers in mathematics has not always been as successful as it might be. In England, the use of calculators with young children has been discouraged in the hope that their absence will enable children to build mental arithmetic relationships. Perhaps this attitude has more to do with the misuse of the calculator (for performing calculations without having to think) than with any inherent defect in the apparatus itself. Used well - to encourage reflection on mathematical ideas - the calculator can be very beneficial. (Tall, 2000, p. 212.)

Many limitations of computational representations for mathematical concepts arise from the algorithm's finite nature. Let us consider the example displayed on figure 1. We see the graphs of the functions $f(x)=\frac{1}{x-1}$ and $g(x)=\frac{1}{(x-1)^{2}}$, respectively, both drawn by Maple. Both the functions have a vertical asymptote at $x=1$, but this line only appears on the picture of the graph of $f$. Actually, the software do not identify the existence of the asymptote for either of the functions. The vertical line displayed is drawn due to a careless algorithm that joins all the points, including one to the left of the discontinuity to one on its right, that is, the software considers the line as part of the graph. The same does not occurs in the case of $\mathrm{g}$ because the lateral limits at $x=1$ are both positive. and the joining of the points occurs offscreen. 

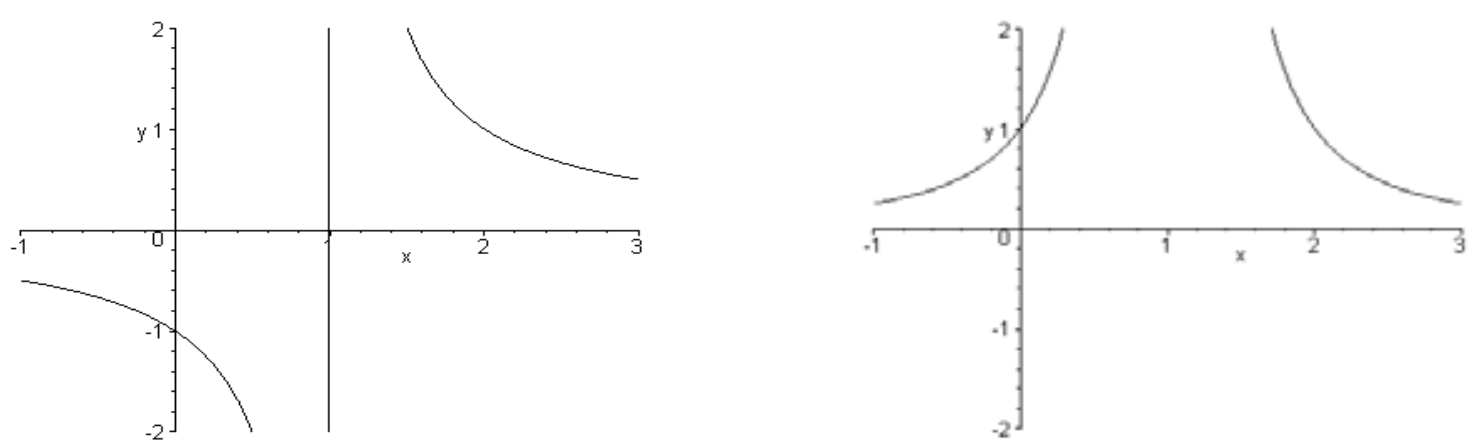

Figure 1. The graphs of $f(x)=\frac{1}{x-1}$ (with a 'fake' asymptote) and $g(x)=\frac{1}{(x-1)^{2}}$. To focus more deeply situations as the one described above, we have used the term theoretical-computational conflict to refer to any situation in which a computational representation is apparently contradictory to the associated theoretical formulation (see Giraldo, 2001; Giraldo and Carvalho, 2002).

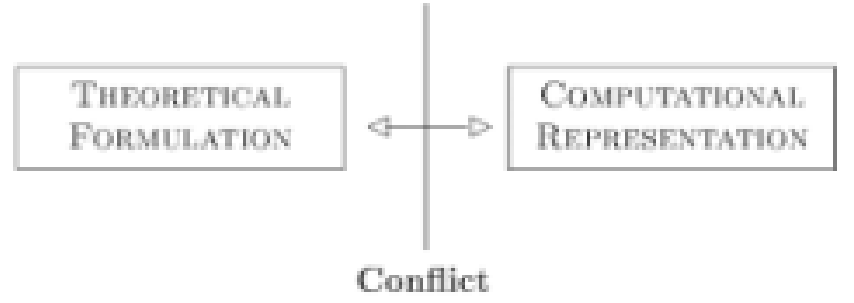

Figure 2. Theoretical-computation conflicts.

We find another example in Mills, Tall and Wardle (1990). The authors report a classroom computational experiment with the quartic equation

$$
x^{4}+2.88 x^{3}-19.23 x^{2}-36.11 x+91.56=0
$$

which has two roots very close together. As a result, floating points errors led to a series of unexpected outputs, among which is a picture displaying more than one thousand roots for a quartic which should have at most four.

A remarkable example of theoretical-computational conflict is shown on figure 3 . The figure displays the process of local magnification of $y=2 x^{2}$, around the point $x_{0}=1$, performed by Maple. Since the curve is differentiable, it should acquire the aspect of a straight line when highly magnified. Rather, due to floating point errors, for very small values of graphic windows ranges (on orders lower than $10^{-6}$ ) it looks like a polygon.
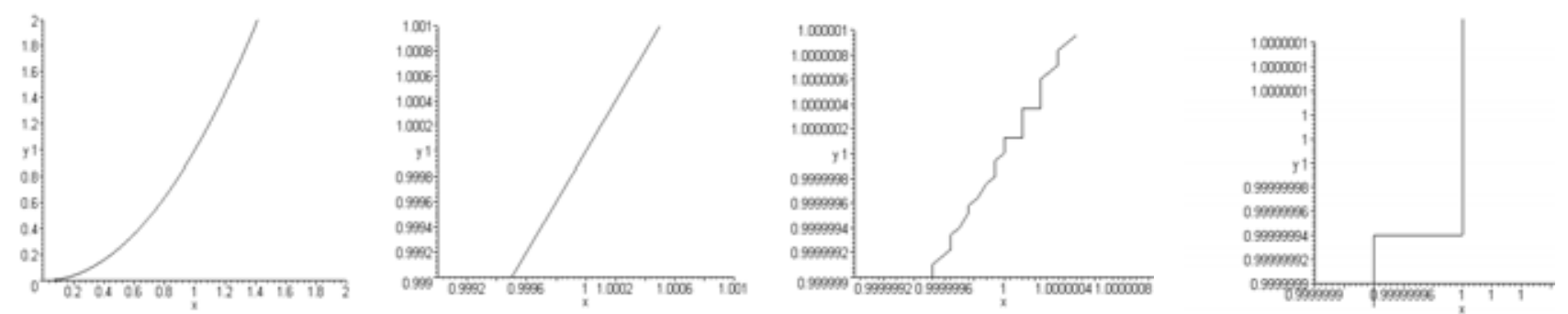

Figure 3. A theoretical-computational conflict on the local magnification process. 
In our own interpretation, the narrowing effect observed on Hunter, Monaghan and Roper's experiment was due not to the occurrence of theoretical-computational conflicts, but, on the contrary, to their absence. Overuse of computational environments - especially when not associated with other forms of representation may contribute to shaping of the conception that the limitations of the representation are characteristics of the mathematical concept itself, leading therefore to the development of narrowed concept images. In fact, Sierpinska observes that:

Many different representations of functions are used, of which tables, graphs and analytic formulae are the most widely known and used, at least at school. Awareness of the limitations of each of the representations and of that they represent one and the same general concept are certainly fundamental conditions of understanding functions. (Sierpinska, 1992, p.49)

We hypothesize that, within a suitable approach, in which theoretical-computational conflicts are emphasized, rather than avoided, the cognitive role of inherent characteristics of each form of representation may have a positive conversion - they may contribute not to the narrowing, but to the enrichment of concept images. On that count, we aim to investigate how theoretical-computational conflicts act on the development of learners' concept images.

\section{A CASE STUDY}

To investigate this hypothesis, we have observed a sample of six first year undergraduate students in Brazil (aged 17 to 20), in twelve individual interviews in which they dealt with situations of (potential) theoretical-computational conflicts. Global results are currently been analyzed. In this section, we will present results of one of those interviews. We will use pseudonyms for the participants: Antônio, Carlos, Francisco, Júlio, Marcelo and Tiago. In this interview, the participants were given two different representations for the function $h(x)=\sqrt{x^{2}+1}$ : the algebraic formula and the graph sketched by Maple for $(x, y) \in[-100,100]^{2}$ (figure 4).

Due to the choice of the graphic window, the curve

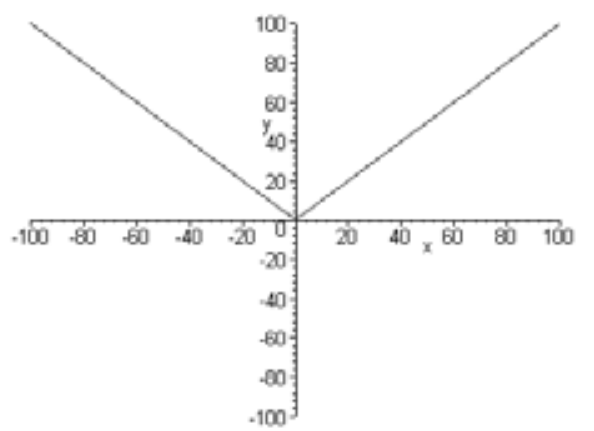

Figure 4. The graph of $h(x)=\sqrt{x^{2}+1}$, for $-100 \leq x \leq 100,-100 \leq x \leq 100$. acquired the aspect of two rays with ends at the origin (in fact, its inclined asymptotes). The conflict here is between the curve displayed on screen, which seemed to have a "corner", and the algebraic expression, which suggested it was differentiable, as figure 5 illustrates. Students were free to manipulate the software as they wanted. Each was asked:

You see on computer's screen the graph of the function $h(x)=\sqrt{x^{2}+1}$, for $-100 \leq x \leq 100$ and $-100 \leq x \leq 100$. Do you think it has a derivative? 
Algebraic Representation: $h$ can be differentiated.

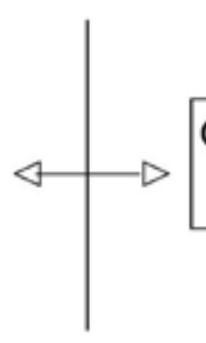

Conflict
Computational Representation: $h$ seems to have a corner.

Figure 5. The potential conflict in the proposed question.

We will now report each participant's strategy on investigating the question (translated from Portuguese). Strategies will be summarized with the help of a diagram, shown on figure 6. The continuous boxes represent the question: Does $h$ have a derivative; and its possible answers: $h$ has a derivative or $h$ doesn't have a derivative. The dashed boxes represent the two given representations for $f$ : computational and algebraic. The arrows indicate the interviewee's actions and are enumerated in chronological order. The boldface arrow indicates the decisive action, that is, the one which led to final conclusion; and the dashed arrow indicates the moment when each participant realises the theoretical-computational conflict.

\section{Antônio}

Antônio claimed immediately that $h$ has a derivative:

Antônio: I think it's differentiable. Look, [points the formula] I see no reason why it shouldn't be. This curve, if you look carefully [points the screen] it meets the other one smoothly, not abruptly at a point, you know? ... Look at here [at the screen], without investigating algebraically, it seems... It's kind of perfect, we can see. But, I can't be based on that, and say: oh, here it looks like a tiny curve, then it is. No! Sometimes, what seems to be is not. So ... here it's the point 0 , right? So, at the point 0 it' 11 be $\left(x^{2}+1\right)^{-1 / 2} \cdot 2 x$, then it' 11 be $0 . .$. So I know it has a derivative.

\section{Francisco}

Francisco started by saying:

Francisco: Looking at it, I think it doesn't have a corner. Then, it'd have a derivative. Now, I want to understand, reasoning ... algebraically why it won't have a corner.

He proceeded:

Francisco: For example, if you made $\sqrt{x^{2}}$, it'd be $|x|$. It'd have a corner. But you've put +1 there, you can't take it off the square root completely, right? ... Visually it isn't a corner, then, it'd have a derivative. I'm speaking in visual terms. Now, let's speak algebraically. Indeed, algebraically, if you differentiate, you'll manage to derive, then, it's differentiable. ... Can we 
zoom in here? [zooms in.] Yes, it looks like a parabola. Zooming in there, you see clearly how it's differentiable.

After concluding about the differentiability of $h$, Francisco spontaneously went on studying the function. He commented:

Francisco: That would be a good question. It looks like a [straight] line, or is it a line? ... I know it has a derivative! I'll try to derive it to see if it is a line or not. [calculates the derivative] Look! This function will have a different slope for each point. It's not like the modulus function, which doesn't have a derivative at 0 , but has the same derivative at the positive side of $x$ and the same one at the negative side for all the points. This function is different, it will be close to the modulus function at $+\infty$ and $-\infty$. It will be close, but for each point it will have a different derivative. So, it looks like a line, but is not a line.

\section{Carlos}

Carlos started by saying:

Carlos: $\quad$...Based on the visualization ... the rough visualization, at that point where it's doing the bifurcation, initially we could say that at that point it's not differentiable. ... The computer ... doesn't have that accuracy to show, here at the point $0 \ldots$ whether it's straight or that ' $\mathrm{V}$ ' from the shape of a corner.

When we asked if he was sure, he replied:

Carlos: $\quad$... We'd evaluate, at that point, the lateral limits, from the left and from the right. Then, in this case, if the limits were different, then at the point it wouldn't have a derivative.

On trying to evaluate the lateral limits, Carlos observed:

Carlos: $\quad$ The point would be 0 . So, the function $f(0)$, would be equal to ... the square root of 0 squared plus 1. $f(0)$ would be equal to 1 ... It doesn't match.

[...] Actually, here you're using a very large interval, from -100 to $100 \ldots$. I'd have to make a better approximation of the graph, shrink the interval, to verify if the $y$ coordinate of $x=0$, would be 0 . [zooms in] Ah! Making an approximation, it's almost ... parabolic. It's a curve. Then, based on the... zoom I made on the graph, we start ... we have the impression that, as it's making a curve, it's not a corner, then it has a derivative at that point. 

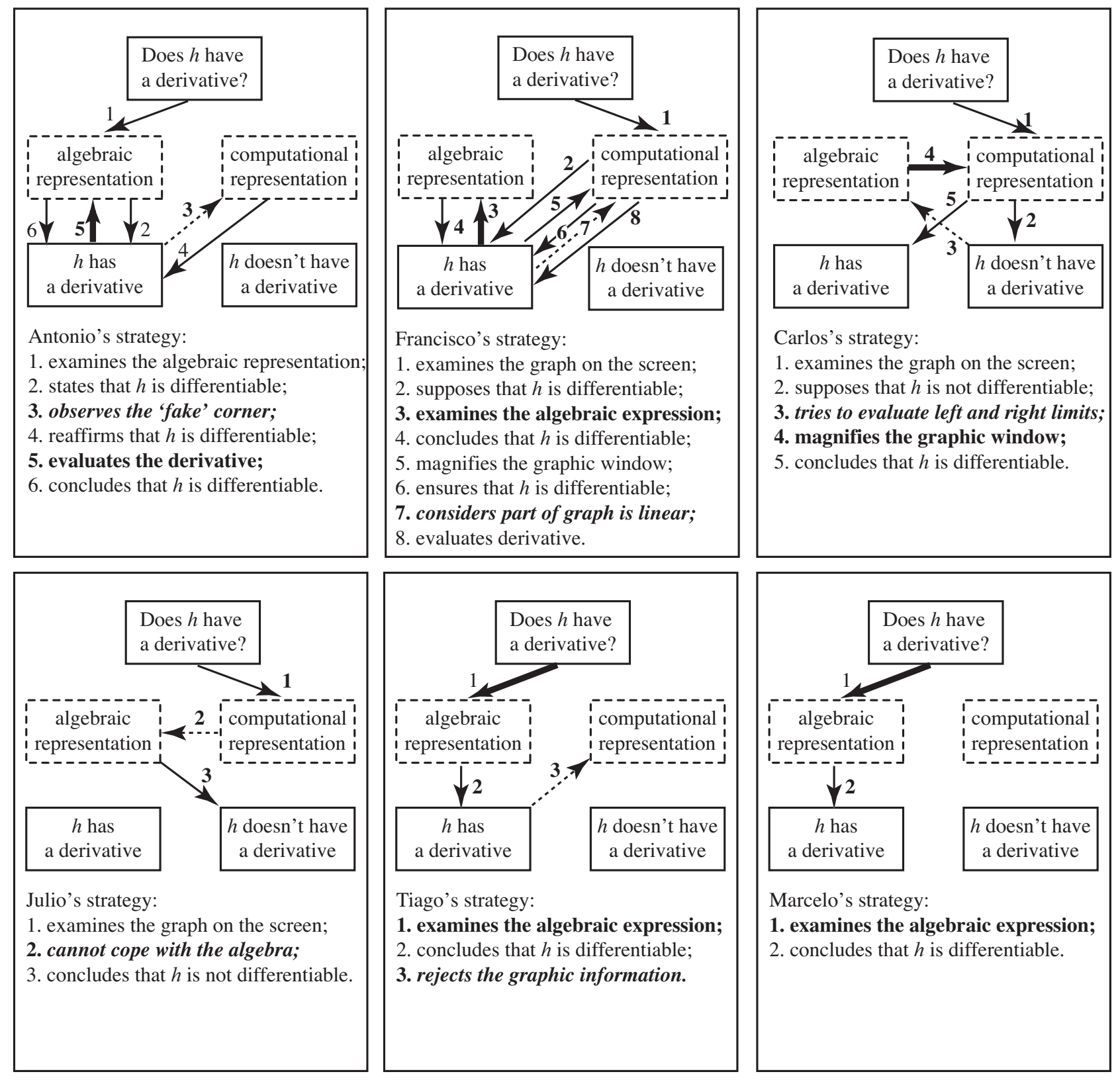

Figure 6. Participants' strategies on investigating the differentiability of $h$

\section{Júlio}

Júlio answered the question:
Júlio: $\quad$ Looking at it, I don't know if it's curve or not. I think that it was a $2^{\text {nd }}$ degree thing, then you've taken the square root off, then the degree became 1. It'll tend to be straight lines. ... As I'll take the root off, when I take off the root it'll be the modulus of the thing which will come off. Then, the function has a corner.

Júlio tried to eliminate the square root by manipulating the function algebraically. After extensively trying, he still did not manage to do so, but even then he stuck with the opinion that $h$ would not be differentiable because a modulus would come out from the square root elimination. 


\section{Tiago}

Tiago was very straight in his answer:

Tiago: I'll treat the square root as being kind of $x^{1 / 2}$. So, in this case, you'd do a power of a power, you'd have a polynomial. So, by the polynomial rule, you'd have the derivative.

When asked about the graph, he replied:

Tiago: It seems not to have a derivative at 0 , I think. ... But this graph is just a ... kind of an allegory. We have to think of the function, purely.

\section{Marcelo}

Marcelo answered simply by saying that the function had a derivative. When asked why by us, he replied:

Marcelo: $\quad$... Because I imagined. ... I've derived other ones ... with something under the root. ... So, I imagined this one could be too. Just that.

We then asked if, in his opinion, the graph was coherent with his answer. He commented:

Marcelo: ... I had not paid any attention to the graph. Just now I noticed it. ... So what?

\section{DISCUSSION}

The data reveals a spectrum of performances. Antônio is highly fluent with the ideas, can see the function is differentiable symbolically, and visualises the essential ideas without using the computer. Francisco can also see that the function is differentiable symbolically, expects it to be smooth at the origin and zooms in to confirm this. He even goes beyond the problem to note that, to the left and right of the origin, the curve looks straight and checks that it cannot actually be linear. Carlos, on the other hand, first suspects from the picture that the function is not differentiable at the origin, attempts to differentiate the expression and fails. He then realises $h(0)$ is 1 , not 0 , and this causes him to zoom in to look at the local behaviour and to conclude that $h$ is differentiable. Júlio is deceived by the picture into thinking it is not differentiable, but cannot cope with the algebra and so accepts the pictorial evidence. Tiago and Marcelo do not use the computer. Tiago differentiates symbolically and correctly concludes that the function is differentiable. Marcelo, on the other hand cannot differentiate the expression, yet believes it is differentiable.

Looking in more detail at each individual, we find that Antônio concludes quickly that the function is differentiable, and therefore it would look smoother if the picture were zoomed in. He does not even need to change the graphic window to be sure. He realises the conflict and understands its source almost immediately. His awareness of the device's limitations prevents him from being misled by the picture displayed on the screen; he even remarks that the conclusion about the problem cannot be based on 
that. Thus, in spite of the fact that he rapidly resolves the conflict, he has experienced it, and it has acted to support his previous knowledge.

Francisco and Carlos both recognize the theoretical-computational conflict. Francisco undertakes flexible connections between computational and algebraic representations in the course of the interview. His conclusion about the differentiability of $h$ is grounded on the algebraic representation - he argues that it is differentiable by using the rules of differentiation. Furthermore, he makes use of the computational representation, zooming in on the graph, to build up a broader understanding of the function's local behaviour. However, the point to underline is that Francisco spontaneously goes further, after establishing the answer for the initially proposed question. He formulates then another question himself, referring to the two parts of the graph to the left and right of the origin: Is it really a straight line or does it only look like a straight line? In the investigation, another cognitive unit is triggered: If the derivative is not constant, then the primitive function is not a straight line. The formulation of the question, which ended up by activating a new cognitive unit, was motivated by a conflict - the graph, as seen on the screen, did not match with the given algebraic expression (figure 7). Carlos' final conclusion was also motivated by a facet of the conflict situation: a mismatch between the evaluation of the function and its visualization on the screen.

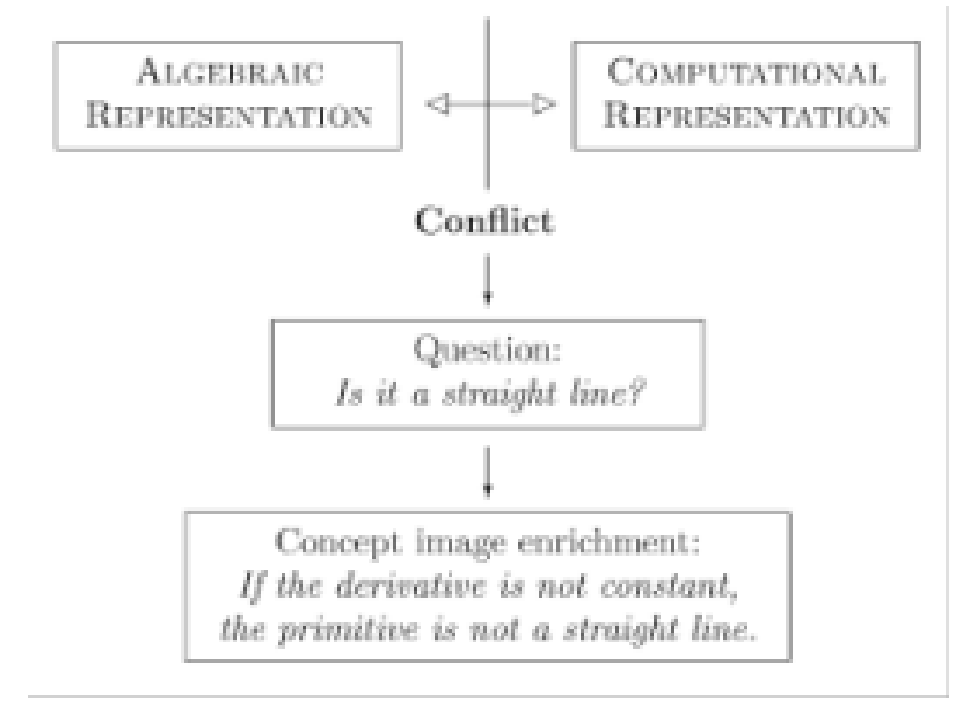

Figure 7. A conflict in Francisco's reasoning

On the other hand, the conflict motivates Júlio to carry out a deeper exploration of the proposed problem. However, from then on, he goes for an exclusively algebraic reasoning, dominated by the idea that he should somehow eliminate the square root. That idea led him to a mistaken conclusion: that the function was not differentiable.

Tiago and Marcelo's behaviours present similarities to each other, since they do not refer to the computer in the course of the interview, unless when requested to do so. Nevertheless, other results in the wider study (currently being analyzed) suggest that such similar behaviour is associated with quite different mental attitudes. Tiago consistently shows an almost exclusive trust on the algebraic forms of representation; 
the computer seems to be irrelevant to him. Marcelo shows resistance towards the computer, in the sense that he avoids dealing with the device whenever he can. As a result, theoretical-computational conflicts often constitute a huge obstacle.

Summarizing the detail, five out of six are affected in some way by the computationaltheoretical conflict. Antônio sees it and resolves it immediately without needing to use the computer. Francisco also quickly resolves the problem, but goes on to use the computer to explore the behaviour away from the origin. Carlos and Julio are both affected by the visual picture and sense the conflict which Carlos is able to resolve using the computer, but Julio is let down by his weakness in algebraic manipulation. Tiago and Marcelo have little interest in the conflict and both work successfully with the algebra. When reminded of the computer picture, Tiago sees there is a problem but rejects it as being an inadequate representation compared with the algebra; Marcelo has no interest in the computer picture.

The results of the experiment suggest that theoretical-computational conflict plays distinct roles for different students. Some, like Francisco, Carlos and Júlio, look first at the picture on the screen; others, like Antônio, Tiago and Marcelo focus first on the algebra. The conflict may either immediately solved (Antônio); barely noticed (Tiago and Marcelo); or may stimulate a deeper exploration, which may or may not lead to the mathematically correct answer (Francisco, Carlos and Júlio).

Perhaps, other conflict situations may act in a different fashion for the spectrum of students. Indeed, this is a research question to be investigated. Their strategies and conclusions are related to specific conceptions and attitudes. Júlio's conclusion is a result of his way of understanding and dealing with algebraic expressions, as he insistently sticks to a certain procedure. Tiago's attitude seems to be associated to the conception that a mathematical object is perfectly defined by its algebraic representation. In fact, he suggests that he thinks of the function purely, by focusing on its formula, so any feedback arising from the computer would be useless for him. For Marcelo, the computer constitutes an actual barrier, which inhibits him from looking through the device and grasping its potentiality to provide useful information.

Our ongoing research continues to study how particular aspects of the concept may be highlighted by suitably designed conflict situations (not necessarily involving computational environments), and hence support each student in the maturation of subtle features in their concept images. Of course, we wish to encourage the building of a richer concept image, which happens in various ways with different students. Our wider goal is to investigate students' mental attitudes associated with different behaviours and triggered by theoretical-computational conflict situations. We aim to comprehend if and how the approach to the concept of derivative can be suitably designed to prompt a positive role for conflicts to enrich learners' concept images. 


\section{REFERENCES}

Abrahão, A.M.C.: 1998, O comportamento de professores frente a alguns gráficos de funções $f: \boldsymbol{R} \rightarrow \boldsymbol{R}$ obtidos com novas tecnologias. Unpublished MSc Dissertation, Pontifícia Universidade Católica, Brasil.

Barnard, A.D. and Tall, D.: 1997, 'Cognitive units, connections, and mathematical proof '. Proceedings of the 21 st Annual Conference of the International Group for the Psychology of Mathematics Education, Lahti, Finland, 2, 41-48.

Belfort, E. and Guimarães, L.C.: 1998, 'Uma experiência com software educativo na formação continuada de professores de matemática'. Anais do VI Encontro Nacional de Educação Matemática, São Leopoldo, Brasil, II, 376-379.

Giraldo, V.: 2001, Magnificação local e conflitos téorico-computacionais. Exame de qualificação (upgrade essay), Programa de Engenharia de Sistemas e Computação, COPPE, Universidade Federal do Rio de Janeiro, Brasil.

Giraldo, V. and Carvalho, L.M.: 2002, 'Local Magnification and Theoreticalcomputational Conflicts'. Proceedings of the 26th Annual Conference of the International Group for the Psychology of Mathematics Education, Norwich, England, 1, 277.

Hunter, M., Monaghan, J.D. and Roper, T.: 1993, 'The effect of computer algebra use on students' Algebraic Thinking'. Working Papers for ESCR Algebra Seminar, Institute of Education, London, UK.

Laudares, J.B. and Lachini, J.: 2000, 'O uso do computador no ensino de matemática na graduação'. 23 ${ }^{a}$ Reunião Anual da Associação Nacional de Pós-Graduação e Pesquisa em Educação, Brasília, Brazil, 32-43.

Mills, J., Tall, D.O. and Wardle, M.: 1990. 'A quartic with a thousand roots'. Mathematical Gazette, 74, 339-346.

Sierpinska, A.: 1992, 'On understanding the notion of function'. In Harel, G. and Dubinsky, E. (eds.), MAA Notes and Report Series (pp. 25-58).

Tall, D.O.: 2001. 'Cognitive development in advanced mathematics using technology'. Mathematics Education Research Journal, 12(3), 210-230.

Tall, D.O.: 2000. 'Biological Brain, Mathematical Mind and Computational Computers (how the computer can support mathematical thinking and learning)'. Plenary Presentation for ATCM Conference, Chang Mai, Thailand. Available on www.davidtall.com/papers.

Tall, D.O. and Vinner, S.: 1981, 'Concept image and concept definition in mathematics with special reference to limits and continuity'. Educational Studies in Mathematics, 12, 151-169.

Thurston, W.P.: 1990, 'Mathematical Education'. Notices of the American Mathematical Society, 37(7), 844-850.

Vinner, S.: 1991. 'The Role of Definitions in the Teaching and Learning of Mathematics'. In Tall, D. (ed.), Advanced Mathematical Thinking (pp. 65-81) Dordrecht: Kluwer. 
Vinner, S.: 1983, 'Concept definition, concept image and the notion of function'. The International Journal of Mathematical Education in Science and Technology, 14, 293-305. 